Ambulance Victoria

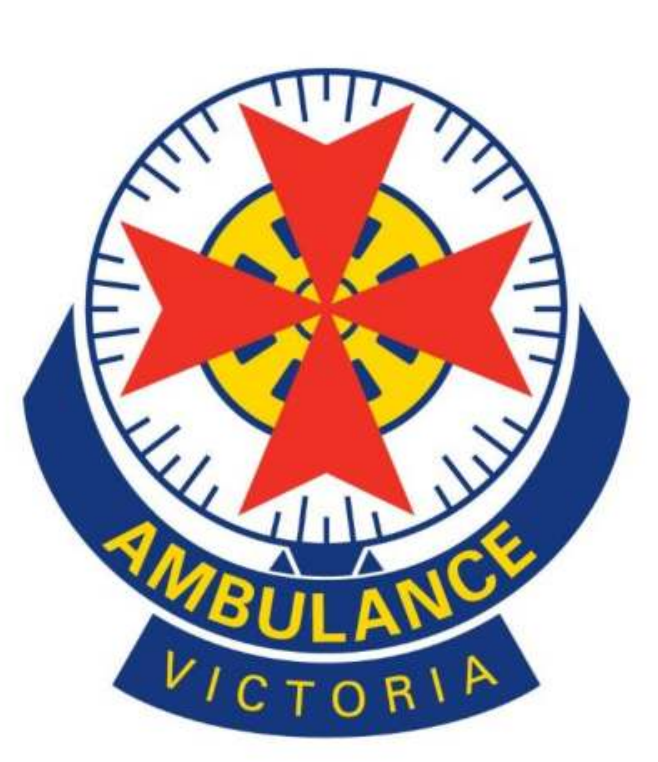

\title{
Paramedic-witnessed paediatric out-of-hospital cardiac arrest in Victoria, Australia
}

\author{
N.Magnuson ${ }^{1}$, Z. Nehme1, 2, S, Bernard ${ }^{1,3}$, K. Smith ${ }^{1}$.
}

${ }^{1}$ Ambulance Victoria, Melbourne, Australia

${ }^{2}$ Monash University, Melbourne, Australia.

${ }^{3}$ The Alfred Hospital, Melbourne, Australia.

\section{Introduction}

Despite paediatric out-of-hospital cardiac arrest (OHCA) being an uncommon event, a small proportion are witnessed by Emergency Medical Services (EMS). It is expected that OHCA witnessed by EMS would have higher survival rates compared to non-EMS witnessed OHCA. However, there have been no previous reports witnessed OHCA. However, there have been no previous reports
describing the characteristics and outcomes of paediatric OHCA according to witness status. This study describes the characteristics and survival outcomes of paediatric OHCA according to witness status in Victoria, Australia.

\section{Methods}

A retrospective observational study of paediatric (age $\leq 16$ years) OHCA cases between 2000 and 2017, with an attempted resuscitation, in the state of Victoria, Australia. Data was extracted from the Victorian Ambulance Cardiac Arrest Registry. Baseline characteristics and survival outcomes were stratified by witness status and reported using descriptive analysis. Adjusted logistic regression analysis was used to compare survival outcomes between witness groups.

\section{Results}

The characteristics of paediatric OHCA are presented in Table 1. Most paediatric OHCA patients were male $(61.38 \%)$, and the median age of the population was 2 years (IQR: 0,10 ).

An initial shockable rhythm was most common in bystander witnessed cases, receiving bystander CPR. In comparison, EMS witnessed cases predominantly presented with pulseless electrical activity (PEA) (65.1\%) as the initial arrest rhythm.
Table 1: Characteristics of paediatric OHCA by witness group

\begin{tabular}{|c|c|c|c|c|c|}
\hline \multirow[t]{2}{*}{ Characteristics } & \multicolumn{2}{|c|}{$\begin{array}{l}\text { Unwitnessed } \\
(n=1063)\end{array}$} & \multicolumn{2}{|c|}{$\begin{array}{l}\text { Bystander Witnessed } \\
\quad(n=516)\end{array}$} & \multirow{2}{*}{$\begin{array}{c}\begin{array}{c}\text { EMS } \\
\text { witnesse }\end{array} \\
\text { (n=96) }\end{array}$} \\
\hline & $\begin{array}{c}\text { No Bystander CPR } \\
\text { (n=231) }\end{array}$ & $\begin{array}{c}\text { Bystander CPR } \\
(\mathrm{n}=474)\end{array}$ & $\begin{array}{c}\text { No Bystander CPR } \\
(n=137)\end{array}$ & $\begin{array}{c}\text { Bystander CPR } \\
(n=266)\end{array}$ & \\
\hline Median Age & 0 & 1 & 5 & 6 & 6 \\
\hline Male & $58.6 \%$ & $63.5 \%$ & $58.4 \%$ & $60.5 \%$ & $64.2 \%$ \\
\hline Metropolitan & $73.5 \%$ & $64.2 \%$ & $71.1 \%$ & $61.7 \%$ & $65.6 \%$ \\
\hline Public location & $5.6 \%$ & $6.3 \%$ & $35.0 \%$ & $41.4 \%$ & $35.4 \%$ \\
\hline Shockable Rhythm & $1.8 \%$ & $3.6 \%$ & $12.9 \%$ & $20.5 \%$ & $2.4 \%$ \\
\hline PEA & $11.2 \%$ & $7.42 \%$ & $28.8 \%$ & $27.0 \%$ & $65.1 \%$ \\
\hline Asystole & $87.0 \%$ & $88.8 \%$ & $57.6 \%$ & $51.7 \%$ & $32.5 \%$ \\
\hline Survival-to-hospital & $13.4 \%$ & $18.0 \%$ & $24.3 \%$ & $29.8 \%$ & $31.3 \%$ \\
\hline $\begin{array}{l}\text { Survival-to-hospital } \\
\text { discharge }\end{array}$ & $4.1 \%$ & $3.8 \%$ & $9.9 \%$ & $16.9 \%$ & $15.4 \%$ \\
\hline
\end{tabular}

Stratified by initial arrest rhythm, survival to hospital discharge was highest in bystander witnessed cases, receiving bystander CPR, given they presented with VF/VT as their initial arrest rhythm. (Figure 1)

Figure 1: Survival-to-hospital discharge stratified by witness group and initial arrest rhythm

SURVIVAL TO HOSPITAL DISCHARGE ACROSS WITNESS GROUP, STARTIFIED BY INITIAL ARREST RHYTHM

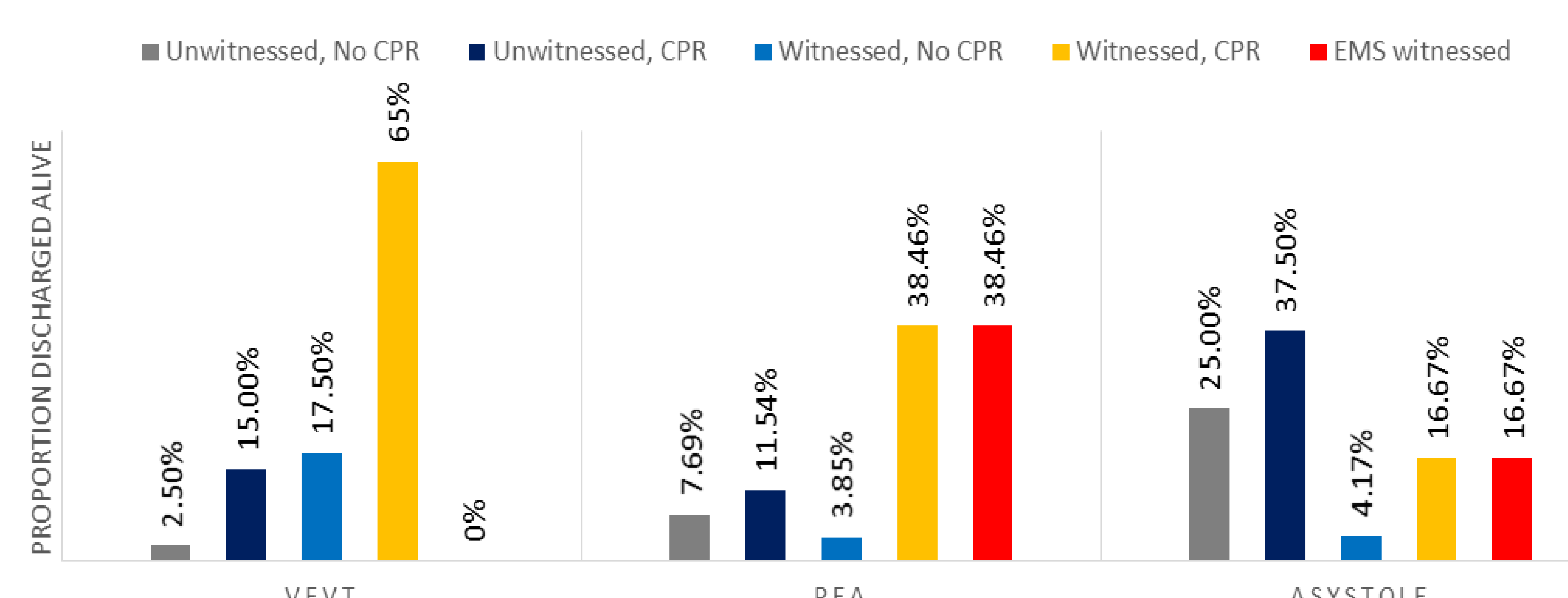

Following multivariable adjustment, the odds of survival-to-hospital was higher in the EMS witnessed cases (OR 1.88, 95\% Cl: 1.03 , 3.43) compared to unwitnessed cases, receiving bystander CPR (Figure 2).

Figure 2: Survival-to-hospital

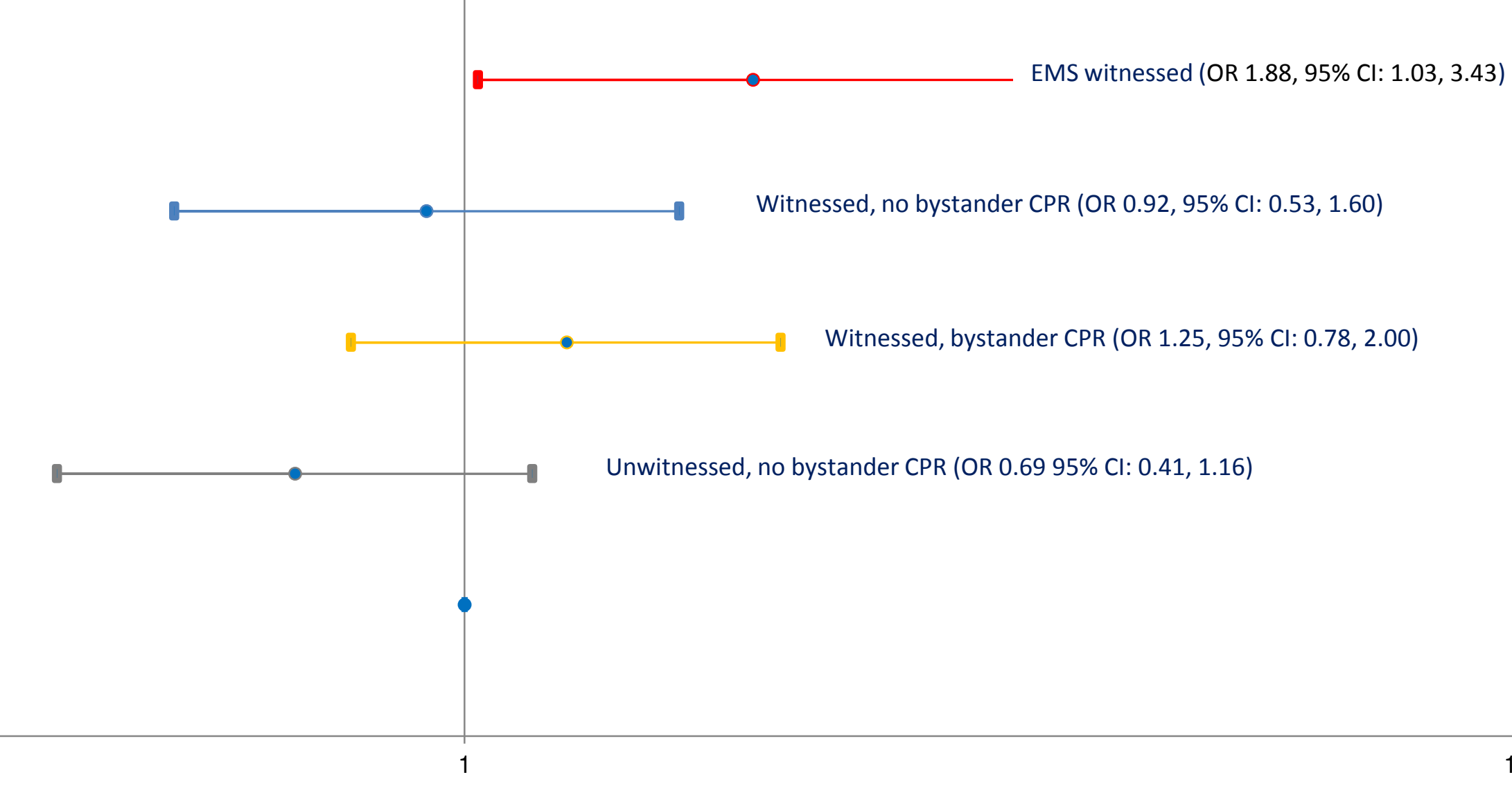

The adjusted odds ratio for survival-to-hospital discharge was highest in the EMS witnessed cases (OR 5.86, 95\% Cl: 2.41 , 14.25) when compared to unwitnessed cases, receiving bystande CPR.

Figure 3: Survival-to-hospital discharge

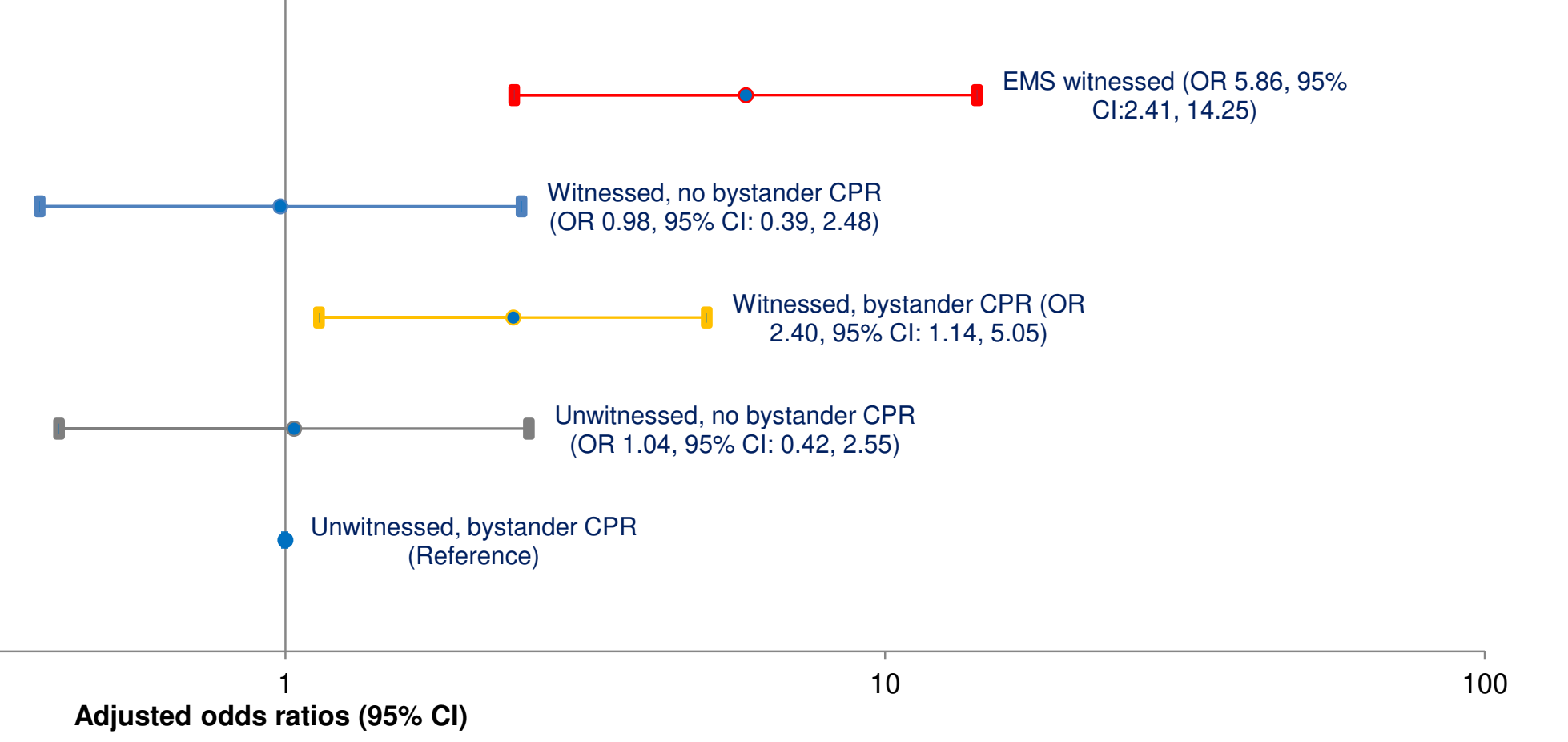

Conclusion

Survival-to-hospital discharge for paediatric OHCA is greatest in the EMS witnessed group compared to bystander witnessed and unwitnessed cases. Additional research is required to examine functional recovery outcomes for paediatric OHCA.

Acknowledgements

Victorian Ambulance Cardiac Arrest Registry 